\title{
Operative travel demand management in the case of evacuations and extraordinary events on a transport network
}

\author{
J. Novák, P. Malina, J. Apeltauer \& R. Čampula \\ Transport Research Center, \\ Division of Human Factors in Transport and Transport Modelling, \\ Czech Republic
}

\begin{abstract}
The base of emergency situation management is a satisfactory information source. The operative center of a traffic police department has basically three ways to gather information about emergency situations: emergency call (which must be verified by police patrol), personal familiarity with the territory (which is highly unlikely) and digital maps. It is obvious that digital maps are the first line of information, and as such, must have maximal efficiency. This paper explains such an information system, which provides aggregate information about an area of interest. The most important information is traffic intensity, capacity of roads, road closures and road limitations (like wide, carrying capacity of the bridge), etc. With these data, the information system can offer detours, with computed travel times. The operations officer can choose one of the offered detours, or only use additional information for his own solution.
\end{abstract}

Keywords: emergency situation, information system, detours, travel times.

\section{Introduction}

Operative travel demand management in case of population evacuation and special events on the transport network aims to remove, or at least reduce, negative impacts on the road and motorway network in the Czech Republic. The project deals with the effective reduction of short-term consequences of road accidents or other traffic limitations as a consequence of floods, wind-fallen trees, etc. It is necessary to determine a reserved capacity of a road segment or junctions and find the best detour alternative. This process includes the use of data on the road and 
motorway network, such as road construction parameters, and dynamic parameters, i.e. traffic volume and modal split.

In order to determine the detour travel time, we use an empiric relationship, which generally expresses the travel times of vehicles. The calculation is based on the increase in free travel time by an increment in relation to volume and capacity. It is an integration of the dynamic phenomena of traffic flow into an ideal and undisturbed vehicle journey on the route. This relationship is complemented by the effect of weather. Regarding the weather effects on road capacity, those are selected for the purpose of the project, which have a significant impact on road capacity reduction and can be monitored by the existing means available in the Czech Republic in real time.

In order to find a detour, the selection criteria identify the road segments which meet sufficient capacity reserve and are available for a given transport mode (e.g. heavy freight traffic). However, detours include junctions, which have a considerable impact on traffic flow, particularly at times of high traffic volume. In order to identify the permeability of junctions according to their individual geometry and traffic volume, we use typical micro-simulation models. Using these models, we are able to estimate the time delay at junctions and specify more accurately the travel time of the designed detour.

The main outcome of the project, which was performed for the Ministry of the Interior, is a tool which can be used by an operation department for crisis traffic management to identify free capacity of roads intended for detours or for the evacuation of the population. This is also related to the optimization of the user interface for the identification of detours, which was developed on the basis of cognitive tests.

\section{Analysis of processes and system modelling}

\subsection{Collection of requirements from users}

A unified development process was used for the designed information system structure. This approach is frequently used in practice and is designed so that the developed system meets the users' requirements and fits to the existing structure of processes. The first step of the unified process is the identification of the socalled 'business processes', or work processes respectively. This process is described in the following section. Once it is clear how the system will be integrated in company processes, it is necessary to specify users' requirements, on which basis the system function will be designed. Based on the interviews with users and the system prototyping, the following abilities were defined as key requirements:

- Minimum demands on the operator in terms of data insertion.

- Minimization of cognitive demandingness of mapping outputs.

- Provision of solutions within minutes.

The designed detour with the possible export of this route into the systems of traffic information for the public was defined as the main outcome. 


\subsection{Business model}

A business model, or a description of company processes, is a key element for the design of the information system. First, it is necessary to document the process into which the system is to be integrated. Then the part of the process which is to be replaced is removed. This process clearly defines the inputs which the system is to process and the expected outcomes which it is to provide. Regarding the OPERA system, the business process is described in Figure 1. With the operative management of special events on the transport network, the whole process is initialized by an emergency call. Based on such an emergency call, the operations officer must localize the place of incident. In the other step, the officer sends a police patrol to such place and the patrol performs the necessary steps to secure and verify the situation, whether it corresponds with the description of the emergency call. Meanwhile, before the police patrol arrives at the place, the operations officer needs to do two things - enter the situation in the information system, so that it may be distributed further and verification. In case there is a potential risk that the road may be completely closed, the officer must prepare detours to guide traffic. At this moment, the OPERA system comes into action, which speeds the process considerably. In the existing situation, the operations officer only has available the topographic map without further information of the road or traffic flow. Therefore, his/her decision-making is rather complicated. In contrast, the OPERA system aggregates all the information into a calculation and provides the operations officer with potential solutions. Furthermore, these solutions are complemented with warnings of critical spots, such as narrowings, bridges, and underpasses. In the case where it is possible to apply alternative traffic management, the OPERA system is able to estimate the delay of vehicles. Based on these designs, the operations officer decides the optimum solution of the situation.

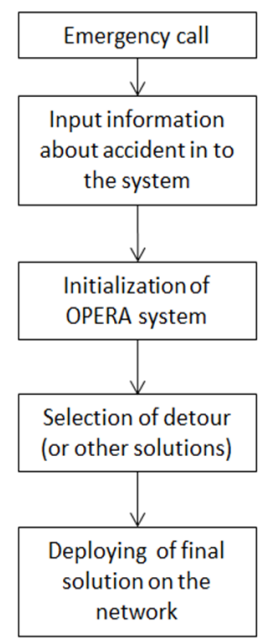

Figure 1: Business model of operative management. 


\section{System structure}

\subsection{Source data}

During extraordinary events, which require evacuation of a population from an affected place or when the transport infrastructure is affected, anomalies on the road network caused by a sudden increase in travel demand occur. The identification of transport characteristics is the basis for effective crisis traffic management. The information provides the operations department of the crisis system with recommendations to eliminate traffic crisis. The characteristics include: daily traffic volume; share of heavy freight traffic; road arrangement; time delays; traffic limitations; weather; whether the road is passable.

A special situation in traffic is considered a situation which leads to a limitation or influence of an otherwise usual traffic situation in the locality. In order to find a suitable design of the detour at the time of its duration, it is necessary to make a record in the information system. After the record is entered, the operation system performs an analysis of the above mentioned characteristics and designs suitable detours (Figure 2).

The capacity of individual elements of the road network is integrated into the system with the use of values according to Czech technical specifications. The capacity of road segments and junctions is based on their geometric parameters. Regarding junctions, capacity models with the use of micro-simulation tools were created. Free capacity of road network segments is set according to the difference in capacity and traffic volume of a given segment. The time delay is calculated using capacity and the existing traffic volume on a segment, which affects the zero time which the vehicle would cover without a limitation. Therefore, other traffic volume on the road is taken into account. Traffic limitations in the form of traffic closures due to road construction or maintenance, or due to a traffic incident, are integrated into the system from the database of the National Transport Information Centre (Národně dopravní informační centrum), which collects such data.

In order to improve the quality of crisis traffic management, it is useful to fill in the data on the location and status of road maintenance vehicles, police patrols, and integrated emergency service vehicles. The source data should have guaranteed their availability and quality. In such case, these data are able to provide materials to a police officer to a sufficient extent. Therefore, the operations officer will have a comprehensive map with estimated normal traffic volumes of Czech road network and potential detours during crisis situation.

\subsection{Data procession and algorithmization}

In the case of a road accident, road traffic on the spot is often limited or the road may be closed. In these situations it is necessary to consider possible detours in terms of their suitability, road arrangement, downhill/uphill gradient, and other transport-engineering parameters. These parameters describe segments and nodes, particularly concerning their technical specification. These are statistical data 


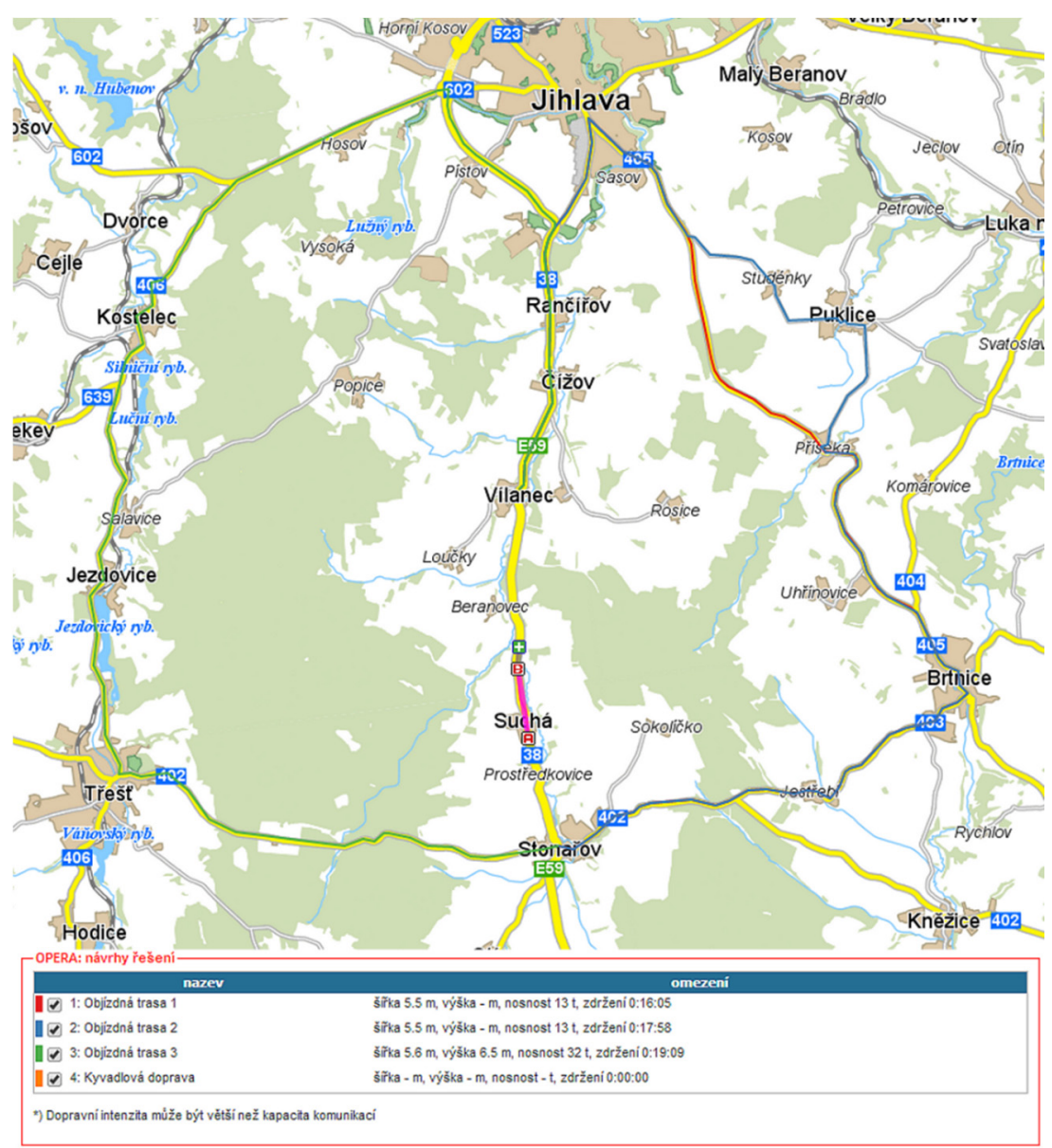

Figure 2: Designed detours in OPERA system.

describing the road network. These data are imported to the system as vector maps of individual segments with descriptive attributes. The source is the road databank of Czech Road Directorate of Roads and Motorways.

The staging requirement for building the system was the maximum speed of feedback. Since it will be used as a tool to guarantee road safety and traffic flow in crisis situations, it is impossible to wait for the calculation of the results for more than several seconds. Therefore, the whole system was divided into two parts, initialization and operation. The conception of the initialization part is based on the calculation preparation of data, which will be only performed during the introduction of new data sets and updates of the road network. The outcome of this stage is optimized and comprised data, which are used as the input for the algorithms to guarantee normal traffic flow. The operation part of the system is not calculation-demanding and is able to serve a sufficient number of requirements for the common traffic. The outcome are the prepared data, which are 
consequently quickly processed. Therefore, the feedback is very fast. Despite the complexity of the whole calculation module, the results are sent back virtually immediately, without delay. Thanks to this, it is possible to deal with the problems on the road network promptly and effectively.

The initialization of the system will be performed with data updates, which take place a maximum of twice a year. Approximately one week of calculation time of the operation server is required for the preparation of all input data. The input data collected form traffic count, Czech Directorate of Roads and Motorways, and geographic information on the road network vary widely, therefore it is necessary to process the information into the required form. The initialization of the system consists of several steps:

- Determination of capacity of road segments: on the basis of geometry, road arrangement, uphill gradient, and heavy freight traffic share, road capacity is determined for every road segment between junctions.

- Determination of traffic volume on road segments: traffic volume, based on regular traffic count, is determined for every road segment between junctions. In the road segments for which the data are unavailable, traffic volume is derived from adjacent roads of similar importance.

- Generating optimized transport network: the input data with road segments contain many attributes which are unused in the described system. The system removes unnecessary attributes and further simplifies the network with an algorithm for linking individual segments. The adaptation of these data leads to a significant simplification and reduction of their amount.

- Generating convertors of segments: a convertor is generated on the basis of spatial information and road segment geometry. With the convertor, it is possible to process data from different sources that enter the system.

- Data storage: all prepared data are stored in a database which is optimized for a fast search. With every future requirement for the calculation of detours, there are many database queries, which need to be dealt with very fast.

- Generating a network graph: a road graph is generated with the use of the optimized road network and parameters of individual road segments. The graph is adapted to the needs of the required outcomes.

Compared to the initialization part of the system, the operating part is computationally very simple. As its input, a calculated road diagram and data from the database of roads are used. These data are further adjusted, based on the time for which a detour is calculated. Road capacity is influenced by the weather, which is also included in the adjustment of initial data. Using the search algorithms in the network diagram, the best detour is subsequently found, which is forwarded to the output together with the time loss.

Determination of time loss on the road segment is derived on the basis of road category. Each category has different traffic engineering parameters and is capable of providing different levels of the quality of traffic service. The model of time losses on roads for emergency traffic management was developed based on the model developed by The Bureau of Public Roads (1964). Travel times in their 
model are derived from unrestricted travel times and time increments affected by capacity and traffic volumes. Free travel time is the time needed to overcome the distance between points $\mathrm{A}$ and $\mathrm{B}$ by the vehicle in unrestricted conditions. However, this is not possible in real life. The calculation is therefore corrected by an increment reflecting the current traffic volume and capacity.

Figure 3 shows the dependence of particular time delays on capacity and traffic volumes, with regard also to calibration parameters of different categories of roads ( $\alpha$ and $\beta$ ).

The model developed by the Bureau of Public Roads considers the time delay according to a two-component exponential model. The first component includes only a time delay in free or zero traffic flow (unaffected by surrounding vehicles). The second component comprises an exponential increase depending on the ratio of traffic volumes and the capacity of the segment. Coefficients $\alpha$ and $\beta$ are parameters of the exponential curve. For example, the velocity $\mathrm{V}=80 \mathrm{~km} / \mathrm{h}$, traffic volume of 1,000 vehicles/h and capacity of 1,200 vehicles/hour give the result of model time delay of about 110 seconds. Thus, the vehicle taking a detour travels for 110 seconds, it needs e.g. 30 seconds more in comparison with the original route where travel time was only 60 seconds. The calculation of travel times also includes the impact of weather on the capacity of roads. It is possible to import data from automatic weather stations into the system, such as: a temperature, precipitation (rain, snow), visibility. It has been found that these characteristics have a considerable impact on the capacity of roads. They may influence the capacity by as much as $5-30 \%$.

\subsection{Time delay at junctions}

The standard CSN 736102 [1] defines the range of acceptable capacity values for 3 types of junctions: controlled by traffic lights, uncontrolled, and roundabouts. This standard specifies the capacity of an uncontrolled junction in the range of 1,500 to 2,000 vehicles per day.

Uncontrolled junctions can further be divided according to their arrangement (number of lanes, width of lanes, geometry, etc.). In order to study the capacity of multiple types of three-arm uncontrolled junctions we used the tool of detailed microscopic simulation of traffic flow.

The capacity of junctions is specified by calculating the capacity of secondary traffic flows. In micro simulations, such distribution of traffic volumes is looked for, which causes extreme situations at the entry from a secondary road. The project tested traffic volume distribution of 111/10-01-01, 111/11-01-01 (extreme, theoretical) and 111/11-11-11 (more realistic) where numeric sequence corresponds to ratios of traffic volumes in terms of: Westbound:Eastbound: Southbound/Thru:Right-Thru:Left-Right:Left (directions/movements).

Micro simulation was performed for 6 arrangement types of uncontrolled junctions.

The standard CSN 736102 [1] defines the capacity of junctions as the boundary for LoS (Level of Service) between the level of E (unstable) and F (over capacity). For an uncontrolled junction it corresponds to a time delay of 45 seconds as the average for all vehicles that passed through a given junction. 
Time delay on roads $\mathrm{CZ}$

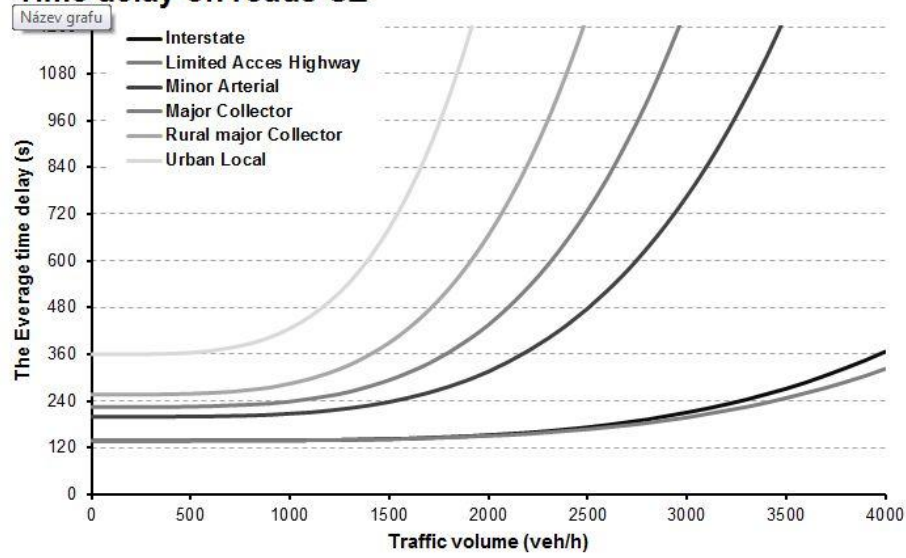

Figure 3: Travel time delays on road segments according to road category.

Figure 4 shows a curve of relationship between average vehicle delays and traffic volumes and is an illustration of one type of the simulated junctions. The darker area corresponds to LoS level of F, i.e. over capacity. The capacity of the junction is given by the junction of the exponential curve showing the distribution of traffic volumes 111/10-01-01 (measured values represented by squares) and the boundary between the coloured areas.

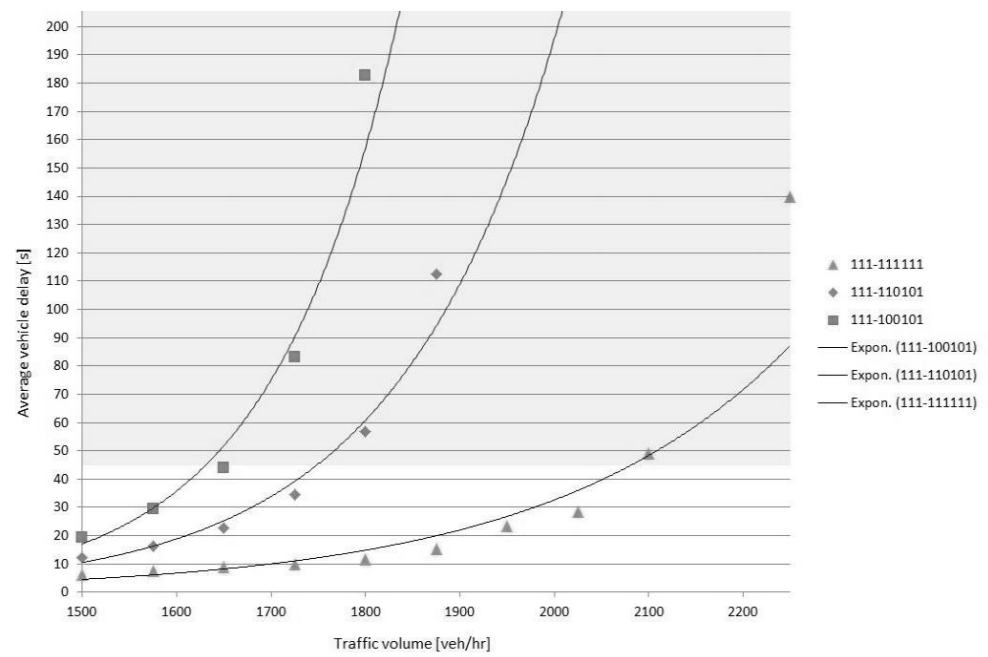

Figure 4: The measured data for one of the types of simulated junctions.

It was found that the arrangement of uncontrolled junctions has a considerable impact on their capacity. It was evaluated that the least capacity arrangement is a junction with one lane at each entrance and an inserted island to protect pedestrians 
on a minor road (SB direction), having a capacity of 1,285 vehicles/hour. It was assessed that the highest capacity junction is the junction with two turning lanes at the entrance of a minor road, which has the capacity 1,773 vehicles/hour.

So the range of values measured using micro simulations on different arrangements of uncontrolled junctions is similar to values given by the standard, but the values are about 200 vehicles lower. It must not be forgotten, however, that our micro simulations examined extreme and theoretical distribution of traffic volumes at junctions. In real traffic, junctions can often accommodate higher traffic volumes.

The project was focused only on measuring three-arm uncontrolled junctions. The tool of micro simulation, however, also has the potential to measure the capacity of other types of junctions. In the project OPERA, the importance of micro simulation of traffic flows has further increased due to its use in the investigation of alternating traffic, where the objective is to determine the capacity of sections with an alternating traffic pattern.

\section{Integration to information structures of the police}

\subsection{Linking with the systems}

Integration of the OPERA system to police managing systems is a necessary step to fully exploit the results of the developed module. Figure 5 presents a simplified scheme of a traffic information system in which OPERA will be included.

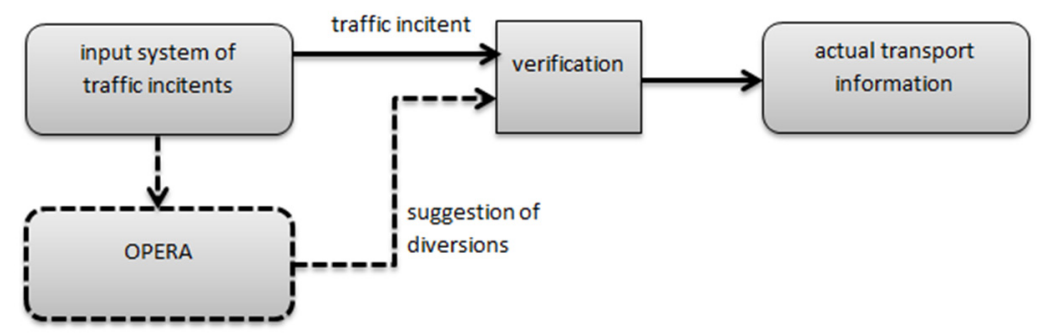

Figure 5: Linking the OPERA system with current systems used by operations department.

Traffic information management is performed using a data input system and information display system. Upon detection of a traffic accident, the necessary information is entered using the input interface. Then it is reviewed and released for further use. The interface for displaying traffic information includes various data necessary for the proper functioning of the operations department. These in particular include real-time traffic information, whether the roads are passable, movement of police patrols, etc.

The OPERA system will be integrated after the input interface for data entry. In case of the need to solve a traffic diversion, the system will evaluate actual traffic conditions and propose an optimal solution. This solution, along with the 
original information about a traffic incident, will be sent for verification and subsequently released to the public.

This concept allows suspending the operation of the OPERA system if necessary, for example, when updating or upgrading search algorithms, without complicated interference in the overall traffic information system.

\section{Conclusion}

The design, pilot and implementation phases have been completed and it is now possible to proceed to the final phase of testing. It will consist of two parts. During the first part, the system will still work separately from live operations and run on a test database. This part can be seen as quasi laboratory, but will be conducted on a much larger number of users and serve to debug most shortcomings and handle user comments. After this short phase, the system will be implemented in live operation, when the final shortcomings will be removed and user comments handled.

This work was supported by the state budget of the Czech Republic and by the project 'Operative travel demand management in case of evacuations and extraordinary events on transport network', identification number VG20102014008 provided by the Ministry of Interior, Security Research Programme of the Czech Republic in 2010-2015 (BVII/2-VS). Participation at this conference has been supported from the project New Approaches to Transport Modelling (CZ.1.07/2.3.00/20.0227) that is co-financed by the European Social Fund and state budget of the Czech Republic.

\section{References}

[1] Alhassan, H. M., and Johnnie, B. E.: Effect of Rainfall Intensity Variability on Highway Capacity' European Journal of Scientific Research, ISSN 1450216X Vol. 49 No. 1 (2011), pp. 18-27.

[2] Chung, E., Ohtani, O. and Kuwahara, M. (2005a). Effect of rainfall on travel time and travel demand. 5th ITS European Congress, Hannover, Germany.

[3] Highway Capacity Manual. Transportation Research Board, 5th edition (2010), Washington, D.C. 2010. ISBN 0-309-16077-4.

[4] Hogema, J.H. (1996) Effects of rain on daily traffic volume and on driving behaviour. TNO report TM-96-B019, TNO Human Factors Research Institute, Soesterberg, The Netherlands.

[5] Juan de Dios Ortúzar, Luis G. Willumsen, Modeling transport 4th Edition, Pontificia Universidad Católica de Chile, Chile, Luis Willumsen Consultance and University College London, UK, 2011, ISBN 978-0-470-76039-0.

[6] Mansoureh jeihani, Stephen Lawe, JP Connolly, Improving Traffic Assignment Model using Intersection Delay Function, Resource Systems Group, Inc. 55 Railroad Row, White River Junction, VT 05001, Submitted for Presentation and publication 47th Annual Transportation Research Forum.

[7] Czech Standards Institute: ČSN 736102 Designing of roads intersections. Prague 2007, EAN 8590963793252. 